\title{
Gestión estratégica: Liderazgo escolar en las instituciones de educación superior en México
}

\author{
Marcela Reyes Pazos, Universidad Autónoma de Baja California, Baja \\ California, México \\ Raul González Núñez, Universidad Autónoma de Baja California, Baja \\ California, México
}

\begin{abstract}
Resumen: El propósito de ésta investigación es definir el perfil del líder escolar dentro de las instituciones de educación superior en México. La presente investigación es descriptiva, con enfoque teórico, donde se presentan las necesidades que demanda el entorno (estudiantes, sociedad, entre otros.), acerca de los líderes, tales como directivos o docentes, en las instituciones educativas, quienes desarrollan estrategias para la toma de decisiones. Se puntualiza al líder escolar en el proceso administrativo de toda organización de educación superior dentro de la etapa de gestión, especificamente la gestión estratégica que debe llevar a cabo en la organización educativa. Se describen las características reales de los líderes escolares en el contexto mexicano, sus fortalezas, así como debilidades. Se explica el perfil idóneo del líder escolar dentro de las instituciones de educación superior, así como las herramientas con las que debe contar para llevar a cabo un buen liderazgo, enmarcando áreas de oportunidad. También se definen las competencias profesionales que deben desarrollar los docentes y directivos que integran de los centros educativos, haciendo énfasis en la gestión estratégica. Se basa en las investigaciones realizadas por la Organización para la Cooperación y Desarrollo Económico (OECD) en México, en el tema de educación.
\end{abstract}

Palabras Clave: Liderazgo Escolar, Dirección Estratégica, Perfil del Líder, Administración Educativa, Educación Superior

\begin{abstract}
The purpose of this research is to define the profile of the school leader in higher education institutions in Mexico. This research is descriptive, with theoretical approach, which presents the needs demanded by the environment (students, society, among others.), about leaders, such as managers or teachers, in educational institutions, who develop strategies for decision making. It points to the school leader in the management process of any organization of higher education in the management stage, specifically strategic management to be carried out in the educational organization. It describes the actual characteristics of school leaders in the Mexican context, strengths and weaknesses. It explains the ideal profile of the school leader in higher education institutions, and the tools with which you must have to perform good leadership, framing areas of opportunity. It also defines the professional skills to be developed by teachers and administrators to integrate schools, focusing on strategic management. It is based on research conducted by the Organization for Economic Cooperation and Development (OECD) in Mexico, in the field of education.
\end{abstract}

Keywords: School Leadership, Strategic Direction, Leading Profile, Educational Administration, Higher Education

Revista Internacional de Educación y Aprendizaje

Volumen 1, 2013, http://sobrelaeducacion.com/revistas/coleccion/, ISSN 2255-453X

(C) Global Knowledge Academics. Marcela Reyes Pazos, Raul González Núñez

Todos los Derechos Reservados. Permisos: soporte@gkacademics.com

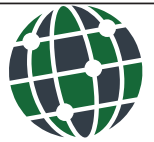




\section{Definiciones conceptuales de liderazgo}

OMO PROCESO EL liderazgo es el uso de la influencia no coercitiva para dirigir y coordinar las actividades de los miembros del grupo para alcanzar una meta según Griffin y Moorhead (2010). Como propiedad, es el conjunto de características atribuidas a quienes, según se percibe, utilizan esa influencia con éxito. La influencia, un elemento común de ambas perspectivas, es la habilidad de afectar las percepciones, creencias, actitudes, motivación y/o conductas de otros. Desde el punto de vista organizacional, el liderazgo es vital porque tiene una influencia muy poderosa sobre el comportamiento individual o de grupo. Además, como la meta hacia la que el grupo dirige sus esfuerzos a menudo es la deseada por el líder, puede interferir o no con las metas de la organización.

El liderazgo no comprende fuerza ni coerción. Un gerente que depende solamente de la fuerza y la autoridad formal para dirigir el comportamiento de sus subordinados no ejerce el liderazgo.

De acuerdo con Lussier y Achua (2011), el tema de liderazgo ha generado emoción e interés desde la antigüedad. No existe una definición universal de liderazgo porque éste es complejo y se analiza en distintas formas, las cuales requieren de diversas definiciones. El liderazgo es el proceso de influencia entre líderes y seguidores para lograr los objetivos organizacionales por medio del cambio. Los elemento claves en la definición de liderazgo son; Influencia, líderes y seguidores, objetivos organizacionales, cambio y las personas.

La influencia es el proceso de un líder al comunicar ideas, ganar aceptación para ellas y motivar a los seguidores para respaldar e implementar las ideas por medio del cambio. La influencia es la esencia del liderazgo. La influencia también comprende la relación entre líderes y seguidores. Cuando mencionamos el término líder, aludimos a alguien que puede ser o no gerente. Un líder siempre tiene la habilidad de influir en los demás, un gerente no. Así, un líder no es necesariamente quien tiene algún puesto formal, como el de gerente. Un seguidor, es quien recibe influencia de un líder. El seguidor puede ser o no gerente.

Los buenos seguidores no son "personas sí" quienes sólo siguen al líder sin ofrecer aportaciones que influyan en el líder. Las cualidades necesarias para un liderazgo efectivo son las mismas que se requieren para ser un seguidor efectivo. Los líderes efectivos, influyen en los seguidores para pensar no sólo en sus propios intereses sino también en el de la organización, por medio de una visión compartida. El liderazgo se presenta cuando los seguidores son influidos para hacer lo que es ético y benéfico para la organización y para ellos mismos.

Influir y establecer objetivos se relaciona con el cambio. Las organizaciones necesitan cambiar en forma continua adaptándose al entorno global de rápido cambio. El liderazgo abarca el influjo en los seguidores para obtener el cambio hacia un futuro deseado por la organización.

Aunque el término personas no se menciona en forma explícita en nuestra definición de liderazgo, después de leer acerca de los demás elementos debe darse cuenta de que el liderazgo trata acerca de liderar personas. La investigación, la experiencia y el sentido común, todo apunta hacia una relación directa entre el éxito financiero de la empresa y su compromiso con las prácticas de liderazgo que tratan a las personas como activos. Según Lussier y Achua (2011), Existe poca evidencia de que ser un gerente grosero y rudo esté asociado con el éxito del liderazgo. Son los esfuerzos colectivos de todas las personas quienes contribuyen, los que hacen que las cosas sucedan. 
Sin embargo, un concepto de liderazgo, que concede cualidades casi místicas al concepto de líder, dando por sentado que sólo los grandes hombres, son dignos de asumir un papel en el poder y la política. El liderazgo se convierte así en un psicodrama en el que una persona, brillante y solitaria, tiene que conseguir el control de sí misma como condición previa para controlar a los demás. Esta expectativa de liderazgo (Harvard Business Review, 1999) marca un profundo contraste con el concepto trivial, práctico y no por ello menos importante, de que el liderazgo es en realidad dirigir el trabajo que realizan otras personas.

\section{Teorías de liderazgo}

De acuerdo con Lussier y Achua (2011), las teorías de los rasgos y conductuales del liderazgo fueron intentos por encontrar el mejor estilo de liderazgo en todas las situaciones. Una teoría de liderazgo es una explicación de cierto aspecto de este último; las teorías gozan de un valor práctico porque se utilizan para entender mejor, predecir y controlar el liderazgo exitoso. Un modelo de liderazgo es un ejemplo para emulación o uso en una situación concreta. La teoría de liderazgo, es el tópico más vasto que explica las variables y los estilos de liderazgo que se utilizarán en una situación de contingencia particular.

Contingencia significa depender. En la Teoría de contingencia y variables del modelo nos dice que los líderes exhiben una gama de conductas en diversas situaciones, ya que el liderazgo está moldeado en gran medida por factores contextuales que no sólo establecen los límites dentro de los cuales interactúan los líderes y los seguidores sino también determinan las demandas y restricciones que confrontan al líder.

Es tan trascendente el liderazgo por contingencia en la economía global (Liderazgo global por contingencias) que nos dice que de un lugar u otro, con diferentes culturas y diferentes estilos de vida se necesita líderes con una apertura internacional y flexible para cualquier lugar.

Robert Tannenbaum y Warren Schmidt citado por Lussier (2011) mencionan que el comportamiento de liderazgo se ubica sobre un continuo que abarca desde el liderazgo centrado en el jefe al liderazgo centrado en los subordinados. Este se enfoca en quien toma las decisiones señalando que la fuerza debe de estar basada en el jefe, los subordinados y la situación. El modelo continuo de liderazgo se utiliza para determinar cuál de los siete estilos. Existen tres fuerzas o variables que debe considerar un líder:

Jefe. La personalidad y el estilo de comportamiento preferido al líder; basado en la experiencia, la expectativa, los valores, antecedentes, conocimiento, percepción de seguridad y confianza en los subordinados, se consideran para elegir el estilo del liderazgo. Con base en la personalidad y el comportamiento, algunos líderes tienden a ser más autocráticos y otros más participativos.

Subordinados. El estilo preferido de los seguidores se funda en la personalidad y el comportamiento. Entre más dispuestos y capaces estén los seguidores a participar, se debe utilizar más libertad de participación y viceversa.

Situación (tiempo). Las características del entorno, como el tamaño, la estructura, el clima, las metas y la tecnología de la organización se deben considerar al elegir un liderazgo.

El modelo de liderazgo trayectoria-meta se utiliza para elegir el estilo de liderazgo (directivo de apoyo participativo u orientado a los logros) apropiado a la situación (subordinado y entorno) para maximizar tanto el desempeño como la satisfacción laborales. 
El líder es responsable de incentivar la motivación de los seguidores para alcanzar metas personales y organizaciones.

La motivación aumenta conforme aumenta la trayectoria de los seguidores para alcanzar recompensas que están disponibles e Incrementar las recompensas que el seguidor valora y desea.

\section{Estilos de liderazgo}

- Liderazgo directivo (el líder proporciona una alta estructura): es el apropiado cuando los seguidores requieren un liderazgo de autoridad, muestran un locus de control externo y la capacidad de los seguidores es limitada, también es adecuado cuando la tarea del entorno es compleja.

- Liderazgo de apoyo (el líder proporciona una alta consideración): es apropiado cuando los seguidores no desean un liderazgo autocritico muestran un locus de control interno y cuando la capacidad del seguidor es alta, también es adecuada cuando las tareas son simples.

- Liderazgo participativo (incluye la participación de los empleados): es apropiado cuando los seguidores pretenden participar, cuando exhiben un locus de control interno y cuando la capacidad del seguidor es alta; cuando la tarea del entorno es compleja.

- Liderazgo orientado a los logros (establece metas difíciles pero alcanzables): espera que los seguidores se desempeñen al más alto nivel y les recompensa por hacerlo, manifiesta un locus de control externo y cuando la capacidad de los seguidores es alta; cuando la tarea del entorno es simple, la autoridad es enérgica y la satisfacción laboral de los compañeros de trabajo es alta o baja.

El modelo de liderazgo normativo tiene un árbol de decisión impulsado por el tiempo y el desarrollo, el cual permite al usuario elegir uno de cinco estilos de liderazgo (decidir, consultar en forma individual, consultar en grupo, facilitar y delegar) apropiados para la situación a fin de maximizar las decisiones.

El modelo normativo proporciona un conjunto secuencial de preguntas que son reglas o normas a seguir para determinar el mejor estilo de liderazgo en una determinada situación.

Variables del Modelo de Liderazgo Normativo, dentro del marco de referencia del liderazgo por contingencia.

Los Estilos de Participación de Liderazgo, de acuerdo con Vroom citado por Lussier (2011), son cinco, basados en el nivel de participación, y en la decisión por parte de los seguidores. Decidir, significa que el líder toma la decisión solo y la anuncia o la vende a los seguidores. Consultar en forma individual, asegura que el líder indica a los seguidores en forma individual acerca del problema. Consultar en grupo, lo define como que el líder los convoca a una reunión de grupo y señala a los seguidores el problema. El líder sostiene una reunión de grupo y actúa como facilitador para definir el problema y los límites dentro de los cuales se debe tomar una decisión. El líder busca participación y coincidencia acerca de la decisión sin imponer sus ideas. El delegar, deja que el grupo diagnostique el problema y toma la decisión dentro de los límites establecidos. El rol de líder es responder preguntas y proporcionar estimulo y recurso.

Los modelos impulsados por el tiempo o impulsados por el desarrollo con base en la situación son los siguientes: 


\section{Método impulsado por el tiempo}

1. Enfoque. El modelo se relaciona con tomar decisiones eficaces con el mínimo costo. El tiempo es dispendioso, ya que toma más tiempo para que los grupos tomen la decisión que el líder solo.

2. Valor. Se concede valor al tiempo; no así al desarrollo de los seguidores.

3. Orientación. El modelo vislumbra un horizonte a corto plazo.

\section{Método impulsado por el desarrollo}

1. Enfoque. El modelo se relaciona con tomar decisiones eficaces con el desarrollo máximo de los seguidores. Esto vale el costo.

2. Valor. Se otorga calor al desarrollo de los seguidores; no así al tiempo.

3. Orientación. El modelo atisba un horizonte a largo plazo, ya que e desarrollo toma tiempo.

Cabe mencionar que las cuatros teorías del liderazgo presentadas son eficaces en cada situación. Steven Kerr y John Jermier (2006), discutieron que ciertas variables situacionales evitan que los líderes afecten las actitudes y las conductas de los subordinados. Los sustitutos del liderazgo incluyen características de los subordinados, tarea y organización que remplazan la necesidad de un líder o neutralizan el comportamiento del líder.

La Universidad de Iowa, hizo estudios acerca del liderazgo, donde Kurt Lewin y sus colegas reconocieron dos estilos básicos: el autoritario y el democrático. El autoritario es quien asume toda la responsabilidad de la toma de decisiones, inicia las acciones, dirige, motiva y controla al subalterno. Sin embargo el estilo democrático o denominado también participativo, es quien consulta, escucha y analiza las ideas en conjunto con los miembros de la organización, acepta sus contribuciones y cultiva la toma de decisiones en conjunto (Chiriboga, 2010).

Otra de las universidades que realizó estudios sobre liderazgo, fue la universidad estatal de Ohio, la cual define dos estilos de liderazgo, uno enfocado en la consideración y otro en la estructura de inicio. La consideración describe el grado de sensibilidad del líder que respeta las ideas y los sentimiento de sus subordinados y crea una confianza reciproca. La estructura de inicio describe la medida en que un líder se orienta hacia las tareas y dirige las actividades laborales de sus subordinados con miras a alcanzar las metas. (Daft, 2006)

\section{Necesidades de líderes escolares que demanda el entorno}

Menciona Cásares (2001) que la educación es uno de los tres pilares sobre los cuales se construye una nueva sociedad, y que el maestro es fundamental para la transformación educativa; de la misma forma, la relación maestro-alumno-comunidad resulta esencial para la construcción de los sistemas y modelos educativos del siglo XXI.

Según Bazarra (2004) se necesitan profesores con características como la pasión por investigar e innovar, su potencial como buenos comunicadores y buenos escuchadores, con capacidad para elegir y crear mejores estrategias que ayuden a un grupo y a cada alumno a encontrar cauces para comprender y comprenderse mejor, y estar preparados para enseñar 
sobre la vida y el mundo a los futuros gobernantes, los que construirán el mundo en el que nos tocará vivir.

La educación según Cásares (2001) se debe enfocar a la formación esencial y a inculcar los valores fundamentales que garanticen la realización personal y familiar, así como la construcción de comunidades solidarias y la transformación del mundo por medio del trabajo.

Una de las tareas urgentes del maestro menciona Cásares (2001) es rescatar el liderazgo. Se requieren lideres que promuevan la esperanza, la fe y el sentido de superación en los niños; que terminen, desde el primer grado escolar, con la simulación, la mentira, y la corrupción; que combaran la mediocridad educativa que se inicia con la falta de preparación de la clase, de algunos maestros con solapar la falta de estudio y del esfuerzo, con la practica corrupta de copiar en los exámenes y de regalar calificaciones; pero, principalmente, con la simulación educativa que enseña datos y conocimientos mas no enseña a vivir, a comprometerse con los semejantes, con la naturaleza, con la propia superación personal...

Según Rojas (2009) coordinador de la Red de Liderazgo en Educación de la OREALC/ UNESCO Santiago, uno de los problemas actuales de la calidad de la educación es la pérdida de la autoridad del profesor, lo que ocurre por múltiples factores: desde la influencia del medio social en los estudiantes hasta déficits en la formación de los docentes.

"La única manera que tiene un profesor de constituirse en líder es que tenga autoridad moral, que tenga credibilidad, infunda confianza, que los niños y niñas lo respeten, que los jóvenes lo sigan. Sólo así se crearán atmósferas de paz y civilidad”, cuenta Rojas.

Rojas (2009) indica que si se analiza en detalle a los docentes que tienen autoridad en sus aulas, es decir, que muestran "actitudes, comportamientos y rasgos que dan confianza, estabilizan el mundo, crean una atmósfera de cordialidad, paz y civilidad y por esa vía infunden respeto y se les sigue", se podrá constatar que disponen de competencias en dos ámbitos de su ser: el de la construcción de confianza, y el de la creación de estados de ánimo.

Los/las docentes deben ser apoyados y ratificados por los directivos y encargados de la disciplina de las escuelas, mención Rojas (2009). Docentes y autoridades deben respaldarse mutuamente y no debilitar su mandato. Un proceso indispensable para que esto ocurra es el de la coordinación del reglamento, las acciones para sancionar que se pueden ejercer en el aula y el apoyo de inspectores y directivos. Las sanciones (y eventualmente los estímulos) que ofrece el reglamento también son promesas. Son promesas institucionales y la institución tiene que tener la capacidad de hacerlas cumplir de modo consistente. De lo contrario, el reglamento, las autoridades y todos los docentes pierden credibilidad. De allí que un principio básico de revisión del reglamento sea depurarlo de todas aquellas reglas que no se van a poder hacer cumplir.

\section{Liderazgo escolar en instituciones de educación}

El liderazgo educativo en las instituciones de educación superior siempre ha sido un factor importante para analizar en el ámbito internacional. Actualmente, en Universidades reconocidas por su prestigio y calidad a nivel internacional, las estrategias motivacionales juegan un papel muy importante para el éxito de las mismas. De acuerdo con Koontz (2008), el desempeño de una institución depende de la organización, como de los atributos del líder mismo. Si queremos incrementar la efectividad organizacional y del grupo, debemos aprender no sólo cómo capacitar a los líderes con mayor efectividad, sino a cómo construir un ambiente organizacional en el que el líder pueda desempeñarse bien. 
Las principales áreas que distinguen a las mejores organizaciones de líderes globales son: enfoque en el crecimiento y la globalización, dedicación al desarrollo de un banco de talento global, estrategia de negocios vinculada al desarrollo de líderes y el perfil que deben tener.

Recientemente, y en la literatura sobre el liderazgo, se ha hecho la distinción entre aquellos modelos centrados en un liderazgo transaccional que asume que los líderes deben ganar legitimidad para poder ejercer influencia.

El directivo docente es finalmente un factor clave de los cambios y transformaciones de las instituciones educativas, las cualidades personales, su formación y experiencia profesional, sus saberes, desempeñan un papel decisivo en la dinámica institucional y en el logro de la finalidad educativa. De acuerdo a Koontz (2008), administrar incluye realizar tareas directivas con efectividad y eficiencia. Una de las principales funciones se relaciona con dirigir en lo general y liderar en lo particular.

Definitivamente la perspectiva internacional de la educación superior en las universidades siempre ha sido un aspecto clave para analizar y desarrollar de acuerdo a la investigación de Fiedler, citada por Robbins (2009) donde menciona que no hay nada automático o "bueno" en el estilo orientado a la tarea, ni en el orientado a las personas, donde también se hace énfasis a las personas.

\section{Liderazgo escolar en instituciones de educación superior}

Según Leithwood (2009), define al liderazgo escolar como la labor de movilizar e influenciar a otros para articular y lograr las intenciones y metas compartidas de la escuela. Este autor usa el término del líder escolar, a la persona que se encarga de la institución educativa, es decir el director, o también a la persona que dirige a un conjunto de instituciones de educación superior, es decir al rector.

Existe poca experiencia todavía en la formación profesional de directivos escolares. Hace falta crear toda una cultura de formación específica para gobernar con profesionalidad en la educación. No resulta suficiente la buena voluntad y la experiencia, pero también es cierto que lo que se enseña en los MBA (Master Business Administration) no siempre resulta directamente aplicable a la educación. (Mañú, 2009).

En términos generales, se entiende por educación superior el nivel formal de estudios en el cual concluyen todas las anteriores etapas de preparación, además de que se considera a sus egresados capaces para el ejercicio de una profesión determinada. Es la última etapa de educación ofrecida, si se considera dentro de ella a los estudios de posgrado y si se ubica como una modalidad educativa separada de los niveles tradicionales, a la llamada educación permanente o continua. (Rodríguez, 2005)

Algunos autores relacionan el término de liderazgo escolar solo con los directores de las instituciones, sin embargo el liderazgo se da en los distintos niveles de la estructura organizacional. Los rectores, directores, y docentes, son líderes escolares, porque ejercen una influencia en las demás personas. Cada uno desde diferente nivel jerárquico; el rector en el nivel más alto hacia los directores, el director en el nivel medio hacia los docentes, y el docente en el nivel bajo hacia los alumnos, donde todos forman parte de la escuela.

El liderazgo escolar en instituciones de educación superior, a nivel internacional cobra gran importancia. El liderazgo escolar universitario, es un proceso continuo, donde cada uno de los actores debe ejercer influencia hacia los otros, para que se realicen los procesos de manera eficiente y eficaz, y que beneficien a la institución. Si uno de los actores que forma 
parte del proceso, no motiva a los demás a que se realicen las operaciones, difícilmente se podrán lograr los objetivos planeados.

Es importante que el liderazgo escolar, se enfoque también en los docentes de las universidades, porque ellos son quienes tienen más contacto directo con los estudiantes. El liderazgo docente ejerce una gran influencia en los alumnos de licenciatura y posgrado. Un docente con habilidades para motivar e inspirar a los estudiantes, crea a individuos con iniciativa para realizar acciones por el bien de la sociedad.

\section{Liderazgo escolar en instituciones de educación superior en México}

El liderazgo de docentes, directivos y rectores en las universidades en México, es fundamental, porque con él los estudiantes tienen ejemplos a seguir para convertirse también en líderes, quienes son el presente y futuro del país.

En México en el año 2010 existían 112, 336, 538 habitantes, que conforman la población total del país, (INEGI, 2012). Hoy en día la cantidad de alumnos en el nivel de educación superior en México es baja, comparado con otros países. En el año 2010 existen 2, 644,197 alumnos que cursan la licenciatura y 208,225 que cursan el posgrado (SNIE, 2012), representan el $2.54 \%$ de la población total. En el año 2010, el $16.5 \%$ de la población total tiene estudios de licenciatura y posgrado. (INEGI, 2012).El nivel educativo tiene relación con el liderazgo escolar, por que los líderes escolares, como son los docentes, influyen para que el alumno tenga buen nivel de aprovechamiento en la escuela, concluya sus estudios.

El líder escolar, también impulsa a los alumnos con buen aprovechamiento a que busquen una beca para solventar sus estudios, y que el factor económico no sea un impedimento para terminar su licenciatura o posgrado. En las universidades públicas de México, existen becas para estudiar, tal es el caso de BECA PRONABES de la Secretaria de Educación Pública para licenciatura (PRONABES, 2012), o BECA CONACYT del Consejo Nacional de Ciencia y Tecnología para posgrado (CONACYT, 2012), además hay becas propias de la universidad.

La gran mayoría de los directivos universitarios públicos en México acceden al cargo por elección de la comunidad universitaria; generalmente, su desempeño ha sido como profesores en cualquier área del conocimiento, y su formación directiva es escasa o nula. (Bennetts, 2007)

De acuerdo a lo que menciona Bennetts, entonces es fundamental para la institución capacitar a los directivos en temas de liderazgo, para que puedan influir en los docentes como piezas importantes de las universidades, quienes imparten los contenidos de las asignaturas, transmiten valores, hacen que los alumnos adquieran destrezas dentro y fuera del aula, e impulsan a los alumnos a que sean más creativos y competitivos.

En México existen dificultades reales en cuanto al desarrollo de la capacidad del liderazgo necesaria para garantizar el progreso en la escuela. Esto se debe a que no ha habido mucha inversión para asegurar una preparación eficaz o una selección de líderes escolares, ni para clarificar el papel que se espera que desempeñen combinando tareas administrativas y pedagógicas. Si la escuela debe ser el núcleo de la educación, es necesario que las escuelas estén bien llevadas y gestionadas. Para que esto suceda en cada escuela de manera durable, es necesario saber con claridad el papel que debe tener y que expectativas debe cumplir el director como líder escolar. (OECD, 2010) 
Las investigaciones sobre procesos de transformación en centros educativos indican que los directores influyen en el desarrollo de procesos tendientes al cambio y la mejora en las universidades. Actualmente, en el contexto de educación superior pública en México, no se ha construido un modelo que permita identificar si existe relación entre los resultados alcanzados en las dependencias universitarias en términos de su desempeño académico y el liderazgo y la autoridad ejercidos por sus directivos. (Bennetts, 2007)

Actualmente, el papel que juega el director en las escuelas mexicanas se inclina más a favor de la administración que del liderazgo profesional. Los directores afirman que su carga administrativa incluye amplias exigencias burocráticas; llenado excesivo de formas y de trámites de seguimiento a los programas centrales. (OECD, 2010)

México requiere de directores que sean líderes, enfocados en el área administrativa, pero que no descuiden la parte pedagógica, que se acerquen a los estudiantes y conozcan cuáles son sus expectativas y necesidades. En algunas universidades de México los alumnos conocen más a los docentes, que al director o al rector, porque ellos se encuentran ocupados con funciones administrativas y políticas.

Las habilidades que requiere un director son distintas a las habilidades que requiere un docente, por lo que el sistema necesita preparar a los líderes usando estándares como punto de partida. El desarrollo del liderazgo debe ser considerado como un continuum. Esto implica alentar la formación inicial del liderazgo, organizar programas de inducción y garantizar la formación en servicio para satisfacer las necesidades del contexto. (OECD, 2010)

También en México se necesitan docentes que ejerzan su liderazgo, y se interesen por el crecimiento de los estudiantes, no solo que aprendan nuevos conocimientos, sino que adquieran nuevas habilidades; que les ayuden a resolver problemas en cualquier lugar. Se vive en un mundo con cambios constantes a cada minuto, y es importante que los docentes preparen a los futuros profesionistas para adaptarse a las transformaciones que surgen en el contexto.

El líder docente juega un papel importante en la sociedad, porque él es quien está formando a los próximos profesionistas, que en un futuro inmediato serán quienes contribuyan a la misma, y es fundamental que el líder motive a sus seguidores. El líder debe transmitir además valores que requiere la sociedad.

Hoy en día la juventud en México, ha transformado sus valores, que provocan acontecimientos desfavorables. Se manifiesta la generación NINI, de jóvenes que ni estudian ni trabajan (Morera, 2012). Esto origina que ellos busquen otras maneras de utilizar su tiempo, y en la mayoría de los casos se ven inmersos en actos delictivos.

En México, en el año 2010, la situación de los jóvenes que están fuera del sistema educativo es preocupante. Casi 19.2 millones de jóvenes de entre 15 y 29 años de edad (lo equivalente al 66.4. de la población total de esta categoría de edad) no están escolarizados: esta es, por mucho, la cifra más alta en los países de la OCDE y los países asociados. De este grupo, mas de 12.5 millones de ellos (65\%) están empleados y 6.7 millones (el 35\%) son jóvenes que ni estudian ni trabajan.(OECD, 2010)

Se demandan docentes comprometidos que no permitan que los estudiantes deserten la universidad; porque si no estos jóvenes no estudiarán, y por el aumento de desempleo que existe en México, no realizarán nada productivo, como consecuencia estarán en las calles delinquiendo y perjudicando a la sociedad. 


\section{Conclusiones}

En las instituciones de educación superior se sugiere una fusión del estilo de liderazgo de la Universidad de Iowa y el de la Universidad de Ohio. Es decir un líder escolar democrático, enfocado en escuchar las ideas de los seguidores para la toma de decisiones, y también basado en la dimensión de consideración, dando importancia a las relaciones humanas.

El perfil idóneo del líder escolar en México, es el estilo democrático con consideración, pero requiere desarrollar competencias como la investigación, gestión, innovación, comunicación, confianza, autoridad moral, el inculcar valores, promover el sentido de superación en los estudiantes, y apoyo y ratificación por parte de los altos mandos en respetar su autoridad.

En las universidades de México, se necesitan líderes comprometidos con la sociedad, pero es un trabajo en conjunto de rectores, directores y docentes, la capacitación de éstos en temas de liderazgo desde distintos niveles, es fundamental para lograr el cambio. El papel del docente como líder es uno de los más importantes, quien ejerce influencia inmediata en los alumnos. Se demandan líderes docentes que formen profesionistas competitivos, creativos, emprendedores, y con habilidades para adaptarse a cualquier transformación del entorno. 


\section{Referencias}

Bazarra Lourdes, Casanova Olga, García Jerónimo. (2004). Ser profesor y dirigir profesores en tiempos de cambio. Narcea ediciones.

Bennetts Fernández María del Socorro. (2007). El liderazgo transformacional y la evaluación de programas académicos universitarios en México. Educar 40. México.

Cásares Arrangoiz David. (2001). Líderes y educadores: el maestro, creador de una nueva sociedad. Edición Biblioteca presidencial para la paz. Gobierno de la Republica. Ministerio de Educación. Guatemala.

Chiriboga P. Hernán, Calva E. Juan. (2010). Formando agrolíderes: metodología para el fortalecimiento del liderazgo en el sector agropecuario . Instituto interamericano de cooperación para la agricultura.

Daft, Richard. (2006). La experiencia del liderazgo. 3ra.edicion. Editorial Thomson.

Griffin Ricky W., Moorhead Gregory (2010). Comportamiento Organizacional. 9na. Ed. México: Cengage, Learning.

Harvard Business Review (1999). Liderazgo. EdicionesDeusto.

Koontz Harold, Weihrich Heinz, Cannice Mark (2008). Administración. 13 era. Ed. Mc. Graw Hill.

Lussier Robert N., Achua Christopher F. (2011). Liderazgo. 4ta. Ed. México. Cengage, Pearson. Learning.

Mañú Noáin José Manuel. (2009). Manual básico de Direc ción escolar. Dirigir es un arte y una ciencia. Narcea ediciones.

Morera Montes Manuel. (2012). El embrujo de los primogénitos. Editorial Grijalbo.

OECD. Organización para la Cooperación y el Desarrollo Económico. (2010). Mejorar las escuelas estrategias para la acción en México.

Rojas Figueroa Alfredo y Lambrech Nora (2009). Construyendo autoridad moral desde las aulas. Reflexiones y propuestas para la acción. UNESCO. Oficina Regional de Educación para América Latina y el Caribe. OREALC/UNESCO Santiago.

\section{Sobre los Autores}

Prof. Marcela Reyes Pazos: Licenciada en administracion de empresas, maestra en administracion y estudiante de doctorado en educacion. Es profesora de asignatura en la Facultad de Ciencias Administrativas y Facultad de Ciencias Sociales y Politicas de la Universidad Autonoma de Baja California.

Prof. Raúl González Núñez: Licenciado en administracion de empresas, maestro y estudiante de doctorado en administracion. Es coordinador de la carrera de Licenciado en Administracion de Empresas, en la Facultad de Ciencias Administrativas de la UABC. 
